\title{
Optimization of Quadruped Robot Locomotion Gaits Through a Genetic Algorithm
}

\author{
Manuel F. Silva \\ ${ }^{1}$ INESC TEC - INESC Technology and Science (formerly INESC Porto) and, \\ ISEP/IPP - School of Engineering, Polytechnic Institute of Porto, Porto, Portugal \\ mss@isep.ipp.pt
}

\begin{abstract}
During the last years research and development on legged robots has grown steadily. Legged systems present major advantages when compared with "traditional" vehicles, allowing locomotion in terrain inaccessible to vehicles with wheels and tracks. However, its energy consumption still lag being these vehicles, existing several aspects that need to be improved and optimized. One of them regards the parameters values that these machines should adopt to minimize the energy consumption. Due to the large number of parameters involved in this optimization process, one way to achieve meaningful results is using evolutionary strategies. Genetic Algorithms are a way to "imitate nature" replicating the process that nature designed for the generation and evolution of species. The objective of this paper is to present a genetic algorithm, running over a simulation application of legged robots, which allows the optimization of several parameters of a quadruped robot model, for distinct locomotion gaits.
\end{abstract}

Keywords - Legged Robots, Locomotion, Gait, Optimization, Genetic Algorithms

\section{Introduction}

Several walking robots have been developed up to date [1]. Compared with traditional vehicles with wheels and tracks, their major advantage is the fact of allowing locomotion in terrain inaccessible to other type of vehicles, because they do not need a continuous support surface.

Since legged locomotion robots are inspired in animals observed in nature, a frequent approach to their design is to make a mechatronic mimic of the animal that is intended to replicate, either in terms of its physical dimensions, or in terms of characteristics such as the gait and the actuation of the limbs. Several examples of robots that have been developed based on this approximation are discussed by Silva and Machado [1].

However, in the present state of development, there are several aspects that need to be improved and optimized in these machines. With this idea in mind, different optimization strategies have been proposed and applied to these systems, either during its design and construction phases, or during its operation [2].

One possibility makes use of genetic algorithms (GAs) as the engine to generate robot structures. GAs are an alternative way of imitating nature. Animals characteristics are not directly copied but, instead, is replicated the process that nature conceives for its generation and evolution. 

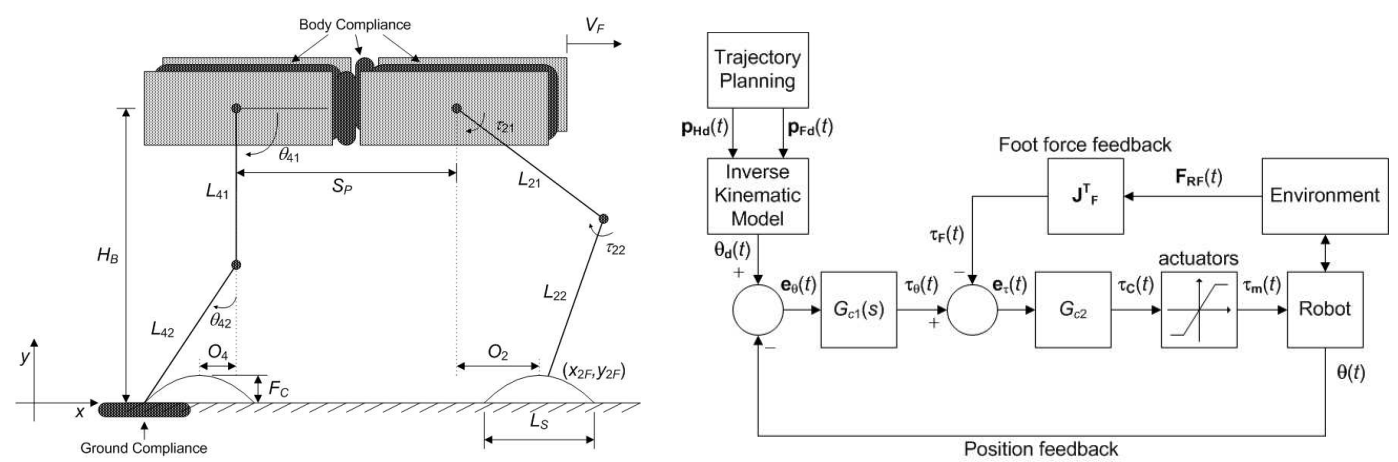

Figure 1: Kinematic and dynamic quadruped robot model (left) and its control architecture (right)

In some cases it is performed a GA modular approach to the robot design $[3,4,5]$. There are also works on which evolutionary strategies are used to optimize the structure of a specific robot $[6,7]$. Other authors proposed the use of GAs for the simultaneous generation of the mechanical structure and the robot controller, for distinct types of robots $[8,9,10,11,12]$.

Bearing these ideas in mind, the objective of this paper is to present a GA, running over a simulation application of legged robots, which allows the optimization of a quadruped robot model parameters, for distinct locomotion gaits often used by animals moving at different velocities.

The remainder of this paper is organized as follows. Section two presents the robot model and its control architecture. Sections three and four present the implemented GA, and some simulation results, respectively. Finally, section five outlines the main conclusions of this study.

\section{Robot Model and Control Architecture}

\subsection{Kinematics and Trajectory Planning}

We consider the model of a legged robot (Figure 1, left) with $n=4$ legs, equally distributed along both sides of the robot body, having each one two rotational joints (i.e., $j=\{1,2\} \equiv\{$ hip, knee\}) [13].

Motion is described by means of a world coordinate system. The kinematic model comprises: the cycle time $T$, the duty factor $\beta$, the transference time $t_{T}=(1-\beta) T$, the support time $t_{S}=\beta T$, the step length $L_{S}$, the stroke pitch $S_{P}$, the body height $H_{B}$, the maximum foot clearance $F_{C}$, the $i^{t h}$ leg lengths $L_{i 1}$ and $L_{i 2}$ (being the total length of each robot leg equal to $1 \mathrm{~m}$ ) and the foot trajectory offset $O_{i}(i=1, \ldots, n)$. Moreover, a periodic trajectory for each foot is considered, with body velocity $V_{F}=L_{S} / T$.

Gaits describe sequences of leg movements, alternating between transfer and support phases. In this work are considered three walking gaits (Walk, Chelonian Walk and Amble), two symmetrical running gaits (Trot and Pace) and five asymmetrical running gaits (Canter, Transverse Gallop, Rotary Gallop, Half-Bound and Bound). These are the gaits usually adopted by animals moving at low, moderate and high speed, respectively, being their main characteristics presented in Table 1.

Given the particular gait and the duty factor $\beta$, it is possible to calculate, for leg $i$, the corresponding phase $\phi_{i}$, the time instant where each leg leaves and returns to contact with the ground and the Cartesian trajectories of the tip of the feet (that must be completed during $t_{T}$ ) [14]. Based on this data, the trajectory generator is responsible for producing a motion that synchronises and coordinates the legs.

The robot body, and by consequence the legs hips, is assumed to have a desired horizontal 
OPTIMIZATION OF QUADRUPED ROBOT GAITS THROUGH A GA

\begin{tabular}{rccccc}
\hline Gait & $\phi_{1}$ & $\phi_{2}$ & $\phi_{3}$ & $\phi_{4}$ & $\beta$ \\
\hline Walk & 0 & 0.5 & 0.75 & 0.25 & 0.65 \\
Chelonian Walk & 0 & 0.5 & 0.5 & 0 & 0.8 \\
Amble & 0 & 0.5 & 0.75 & 0.25 & 0.45 \\
Trot & 0 & 0.5 & 0.5 & 0 & 0.4 \\
Pace & 0 & 0.5 & 0 & 0.5 & 0.4 \\
Canter & 0 & 0.3 & 0.7 & 0 & 0.4 \\
Transverse Gallop & 0 & 0.2 & 0.6 & 0.8 & 0.3 \\
Rotary Gallop & 0 & 0.1 & 0.6 & 0.5 & 0.3 \\
Half-Bound & 0.7 & 0.6 & 0 & 0 & 0.2 \\
Bound & 0 & 0 & 0.5 & 0.5 & 0.2 \\
\hline
\end{tabular}

Table 1: Quadruped gait parameters

movement with a constant forward speed $V_{F}$, being the Cartesian coordinates of the hip of the legs, for leg $i$, given by $\mathbf{p}_{\mathbf{H d}}(t)=\left[x_{i H d}(t), y_{i H d}(t)\right]^{\mathbf{T}}[13]$.

Regarding the feet trajectories, for each cycle, the desired trajectory of the foot of the swing leg is computed through a cycloid function and described by (for leg $i$ ) $\mathbf{p}_{\mathbf{F d}}(t)=\left[x_{i F d}(t), y_{i F d}(t)\right]^{\mathbf{T}}[13]$.

The algorithm for the forward motion planning accepts, as inputs, the desired Cartesian trajectories of the leg hips $\mathbf{p}_{\mathbf{H d}}(t)$ and feet $\mathbf{p}_{\mathbf{F d}}(t)$ and, by means of an inverse kinematics algorithm $\psi^{-1}$, generates as outputs the joint trajectories $\boldsymbol{\Theta}_{\mathbf{d}}(t)=\left[\theta_{i 1 d}(t), \theta_{i 2 d}(t)\right]^{\mathbf{T}}[13]$, that constitute the reference for the robot control system. In this study it is adopted the mammal leg configuration, namely selecting in $\psi^{-1}$ the solution corresponding to a forward knee.

In order to avoid the impact and friction effects, at the planning phase null velocities of the feet are considered in the instants of landing and taking off, assuring also the velocity continuity.

\subsection{Robot Dynamic Model}

\subsubsection{Inverse Dynamics Computation}

The model for the robot inverse dynamics is formulated as [13]:

$$
\boldsymbol{\Gamma}=\mathbf{H}(\boldsymbol{\Theta}) \ddot{\boldsymbol{\Theta}}+\mathbf{c}(\boldsymbol{\Theta}, \dot{\boldsymbol{\Theta}})+\mathbf{g}(\boldsymbol{\Theta})-\mathbf{F}_{\mathbf{R H}}-\mathbf{J}^{\mathbf{T}}(\boldsymbol{\Theta}) \mathbf{F}_{\mathbf{R F}}
$$

where $\boldsymbol{\Gamma}$ is the vector of forces/torques, $\Theta$ is the vector of position coordinates, $\mathbf{H}(\boldsymbol{\Theta})$ is the inertia matrix and $\mathbf{c}(\boldsymbol{\Theta}, \dot{\boldsymbol{\Theta}})$ and $\mathbf{g}(\boldsymbol{\Theta})$ are the vectors of centrifugal/Coriolis and gravitational forces/torques, respectively. The matrix $\mathbf{J}^{\mathbf{T}}(\Theta)$ is the transpose of the robot Jacobian matrix, $\mathbf{F}_{\mathbf{R H}}$ is the vector of the body inter-segment forces and $\mathbf{F}_{\mathbf{R F}}$ is the vector of the reaction forces that the ground exerts on the robot feet, being null during the foot transfer phase.

Moreover, the joint actuators are not considered ideal, exhibiting a saturation, being the maximum torque that each actuator can supply $\tau_{i j M a x}$.

\subsubsection{Robot Body Model}

The dynamic model for the hexapod body and foot-ground interaction (Figure 1) considers a compliant robot body, divided in $n$ identical segments (each with mass $M_{b} n^{-1}$, while making the total mass of the robot equal to $100 \mathrm{~kg}$ ) and a linear spring-damper system is adopted to implement the intra-body [13]. The parameters of this spring-damper system, $K_{\eta H}$ and $B_{\eta H}(\eta=\{x, y\}$ in the 
\{horizontal, vertical\} directions, respectively), are defined so that the body behavior is similar to the one expected to occur on an animal [13].

\subsubsection{Foot-Ground Interaction Model}

The contact of the $i^{\text {th }}$ robot foot with the ground is modelled through a non-linear system (Figure 1) with linear stiffness $K_{\eta F}$ and non-linear damping $B_{\eta F}(\eta=\{x, y\}$ in the horizontal, vertical $\}$ directions, respectively) [15]. The values for the parameters $K_{\eta F}$ and $B_{\eta F}$ are based on the studies of soil mechanics [15].

\subsection{Control Architecture}

The general control architecture of the multi-legged locomotion system is depicted in Figure 1 (right), being $G_{c 1}(s)$ a PD controller and $G_{c 2}$ a simple P controller [15]. The trajectory planning is held in the Cartesian space, but the control is performed in the joint space, which requires the integration of the inverse kinematic model in the forward path. The control algorithm considers an external position and velocity feedback and an internal feedback loop with information of footground interaction force.

\section{Developed Genetic Algorithm}

GAs are adaptive methods which may be used to solve search and optimization problems [16]. By mimicking the principles of natural selection, GAs are able to evolve solutions towards an optimal one. Although the optimal is not guaranteed, the GA is a stochastic search procedure that, usually, generates good results. The GA maintains a population of candidate solutions (the individuals). Individuals are evaluated and fitness values are assigned based on their relative performance. They are then given a chance to reproduce, i.e., replicating several of their characteristics. The offspring produced are modified by means of mutation and/or recombination operators before they are evaluated and reinserted in the population. This is repeated until some condition is satisfied.

\subsection{Structure of the Used Chromosome}

The chromosome used in the developed GA presents 34 genes (i.e., 34 robot parameters). The genes are organized as presented in Table 2: the first gene $\left(L_{s}\right)$ contains information regarding the step length and the last gene $\left(K d_{22}\right)$ contains the derivative gain of joint 2 of the robot rear legs. These values are coded directly into real numbers (value encoding).

\subsection{Measure for the Fitness Evaluation}

For the fitness function is used the mean absolute density of energy per travelled distance $E_{a v}$ [17]. This index is computed assuming that energy regeneration is not available by actuators doing negative work (by taking the absolute value of the power). At a given joint $j$ (each leg has $m=2$ joints) and leg $i$ ( $n=4$ legs since a quadruped is being considered), the mechanical power is the product of the motor torque and angular velocity. The global index $E_{a v}$ is obtained by averaging the mechanical absolute energy delivered over the travelled distance $d$ :

$$
E_{a v}=\frac{1}{d} \sum_{i=1}^{n} \sum_{j=1}^{m} \int_{0}^{T}\left|\tau_{i j}(t) \dot{\theta}_{i j}(t)\right| d t \quad\left[\mathrm{Jm}^{-1}\right]
$$

The performance optimization is achieved through the minimization of the index $E_{a v}$. 


\begin{tabular}{|c|c|c|}
\hline Minimum Value & Variable & Maximum Value \\
\hline $0<$ & $L_{s}$ & $\leq 10 \mathrm{~m}$ \\
\hline $0<$ & $H_{B}$ & $\leq 1 \mathrm{~m}$ \\
\hline $0<$ & $F_{C}$ & $\leq 1 \mathrm{~m}$ \\
\hline $0<$ & $L_{11}$ & $\leq 1 \mathrm{~m}$ \\
\hline $0<$ & $L_{12}$ & $\leq 1 \mathrm{~m}$ \\
\hline $0<$ & $L_{21}$ & $\leq 1 \mathrm{~m}$ \\
\hline $0<$ & $L_{22}$ & $\leq 1 \mathrm{~m}$ \\
\hline $0<$ & $O_{1}$ & $\leq 10 \mathrm{~m}$ \\
\hline $0<$ & $\mathrm{O}_{2}$ & $\leq 10 \mathrm{~m}$ \\
\hline $0<$ & $M_{b}$ & $\leq 100 \mathrm{~kg}$ \\
\hline $0<$ & $M_{11}$ & $\leq 10 \mathrm{~kg}$ \\
\hline $0<$ & $M_{12}$ & $\leq 10 \mathrm{~kg}$ \\
\hline $0<$ & $M_{21}$ & $\leq 10 \mathrm{~kg}$ \\
\hline $0<$ & $M_{22}$ & $\leq 10 \mathrm{~kg}$ \\
\hline $0<$ & $K_{x h}$ & $\leq 10000 \mathrm{Nm}$ \\
\hline $0<$ & $K_{y h}$ & $\leq 10000 \mathrm{Nm}$ \\
\hline $0<$ & $B_{x h}$ & $\leq 10000 \mathrm{Nms}^{-1}$ \\
\hline $0<$ & $B_{y h}$ & $\leq 10000 \mathrm{Nms}^{-1}$ \\
\hline$-400<$ & $\tau_{11 \min }$ & $\leq 0 \mathrm{Nm}$ \\
\hline $0<$ & $\tau_{11 \text { Max }}$ & $\leq 400 \mathrm{Nm}$ \\
\hline$-400<$ & $\tau_{12 \min }$ & $\leq 0 \mathrm{Nm}$ \\
\hline $0<$ & $\tau_{12 \operatorname{Max}}$ & $\leq 400 \mathrm{Nm}$ \\
\hline$-400<$ & $\tau_{21 \min }$ & $\leq 0 \mathrm{Nm}$ \\
\hline $0<$ & $\tau_{21 M a x}$ & $\leq 400 \mathrm{Nm}$ \\
\hline$-400<$ & $\tau_{22 \min }$ & $\leq 0 \mathrm{Nm}$ \\
\hline $0<$ & $\tau_{22 \operatorname{Max}}$ & $\leq 400 \mathrm{Nm}$ \\
\hline $0<$ & $K p_{11}$ & $\leq 10000$ \\
\hline $0<$ & $K d_{11}$ & $\leq 1000$ \\
\hline $0<$ & $K p_{12}$ & $\leq 10000$ \\
\hline $0<$ & $K d_{12}$ & $\leq 1000$ \\
\hline $0<$ & $K p_{21}$ & $\leq 10000$ \\
\hline $0<$ & $K d_{21}$ & $\leq 1000$ \\
\hline $0<$ & $K p_{22}$ & $\leq 10000$ \\
\hline $0<$ & $K d_{22}$ & $\leq 1000$ \\
\hline
\end{tabular}

Table 2: Interval of variation of the 34 genes used in the chromosome 


\subsection{Base Structure of the Developed GA}

The outline of the specific GA is as follows:

1. Start: Generate a random population of $v=50$ chromosomes. The values for the genes that constitute the chromosome are uniformly distributed in the ranges mentioned in Table 2.

2. Simulation: Simulate the robot locomotion for all chromosomes in the population.

3. Fitness: Evaluate the fitness function for each chromosome by computing $E_{a v}$.

4. New population: Create a new population by repeating the following steps:

- Selection - Select the 4 best parent chromosomes according to their fitness. These solutions are copied without changes to the new population (elitism).

- Crossover - Select $90 \%$ of the individuals to be replaced by the crossover of the parents: two random parents are chosen and an arithmetic mean operation is performed to produce one new offspring.

- Mutation - Select $1 \%$ of the individuals to be replaced by mutation of the parents: one random parent is chosen and a small number is added to selected values, to make a new offspring.

- Spontaneous generation - The remaining individuals are replaced by new randomly generated ones (such as in step 1).

5. Loop: If this iteration is the $500^{t h}$ or the GA has converged (the value of the fitness function for the chromosome with the best fitness function is equal to the one that is in the position corresponding to $90 \%$ of the population), stop the algorithm, else, go to step 2.

\section{Simulation Results}

The main objective of this study was to find the optimal values for the robot model and controller parameters, considering that the robot was moving with $V_{F}=1 \mathrm{~ms}^{-1}$, while adopting the gaits Walk (1), Chelonian Walk (2), Amble (3), Trot (4), Pace (5), Canter (6), Transverse Gallop (7), Rotary Gallop (8), Half-Bound (9) and Bound (10).

This study started by determining the optimal values for the robot model and controller parameters, considering a robot moving at $V_{F}=1 \mathrm{~ms}^{-1}$, with the Walk Gait. Running the GA, with the parameters described in subsection 3.3, the algorithm converged to the results given in Table 3.

Analyzing the results presented in Table 3 it should be referred that the length of the upper segment of the leg should be smaller than the corresponding length of the lower segment. In the same way, the upper segment of the leg should be heavier than the lower segment. Finally, the trajectory of the legs must be displaced to the rear of the moving direction, as indicated by the values of the parameters $O_{i}$.

Following, the GA was executed with the same parameters, for the distinct gaits under analysis. The algorithm converged to the results that are described in the sequel. There was one exception: for the Chelonian Walk gait the GA did not converge, although several attempts (distinct runs of the GA) were made.

Figure 2 depicts two charts with the optimum values of the Step Length $\left(L_{S}\right)$ (left) and Body Height $\left(H_{B}\right)$ (right), for the distinct gaits under consideration, determined by the GA. It is seen that the robot should move with a value of $L_{S} \approx 1.1 \mathrm{~m}$, except for the Canter and Bound gaits, for which case it should be $L_{S} \approx 1.3 \mathrm{~m}$. Concerning the value for the Body Height, analyzing the chart presented in the left part of this figure, one can conclude that the robot should adopt a value of $H_{B} \approx 0.8 \mathrm{~m}$. 


\begin{aligned} \hline Parameter & Optimum Value \\ \hline$L_{s} & =1.056 \mathrm{~m} \\ H_{B} & =0.724 \mathrm{~m} \\ F_{C} & =0.076 \mathrm{~m} \\ L_{11} & =0.484 \mathrm{~m} \\ L_{12} & =0.516 \mathrm{~m} \\ L_{21} & =0.425 \mathrm{~m} \\ L_{22} & =0.575 \mathrm{~m} \\ O_{1} & =-0.383 \mathrm{~m} \\ O_{2} & =-0.035 \mathrm{~m} \\ M_{b} & =83.134 \mathrm{~kg} \\ M_{11} & =4.976 \mathrm{~kg} \\ M_{12} & =2.923 \mathrm{~kg} \\ M_{21} & =6.485 \mathrm{~kg} \\ M_{22} & =2.482 \mathrm{~kg} \\ K_{x h} & =79991.055 \mathrm{Nm} \\ K_{y h} & =9084.575 \mathrm{Nm} \\ B_{x h} & =991.235 \mathrm{Nms} \\ B_{y h} & =92.299 \mathrm{Nms} \\ \tau_{11 \min } & =-296.987 \mathrm{Nm} \\ \tau_{11 M a x} & =105.782 \mathrm{Nm} \\ \tau_{12 m i n} & =-136.718 \mathrm{Nm} \\ \tau_{12 M a x} & =145.311 \mathrm{Nm} \\ \tau_{21 \text { min }} & =-287.426 \mathrm{Nm} \\ \tau_{21 \mathrm{Max}} & =115.196 \mathrm{Nm} \\ \tau_{22 \mathrm{~min}} & =-283.489 \mathrm{Nm} \\ \tau_{22 \mathrm{Max}} & =342.611 \mathrm{Nm} \\ K p_{11} & =3012.207 \\ K d_{11} & =789.264 \\ K p_{12} & =4395.400 \\ K d_{12} & =165.975 \\ K p_{21} & =3202.196 \\ K d_{21} & =543.265 \\ K p_{22} & =5429.295 \\ K d_{22} & =156.955 \\ & \end{aligned}$

Table 3: Optimum values for the hexapod parameters while walking with the Walk Gait, being $V_{F}=1 \mathrm{~ms}^{-1}, E_{a v}=500.002 \mathrm{~J} / \mathrm{m}$ and the travelled distance $d=1.281 \mathrm{~m}$. 

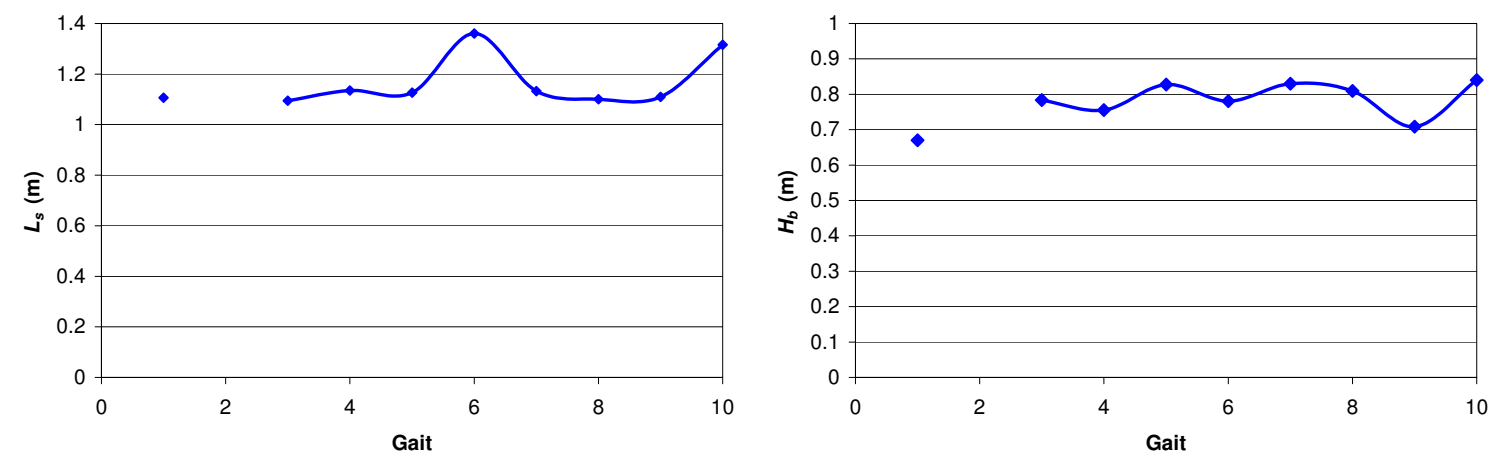

Figure 2: Optimum values of the Step Length $L_{S}$ (left) and Body Height $H_{B}$ (right), for the gaits under study
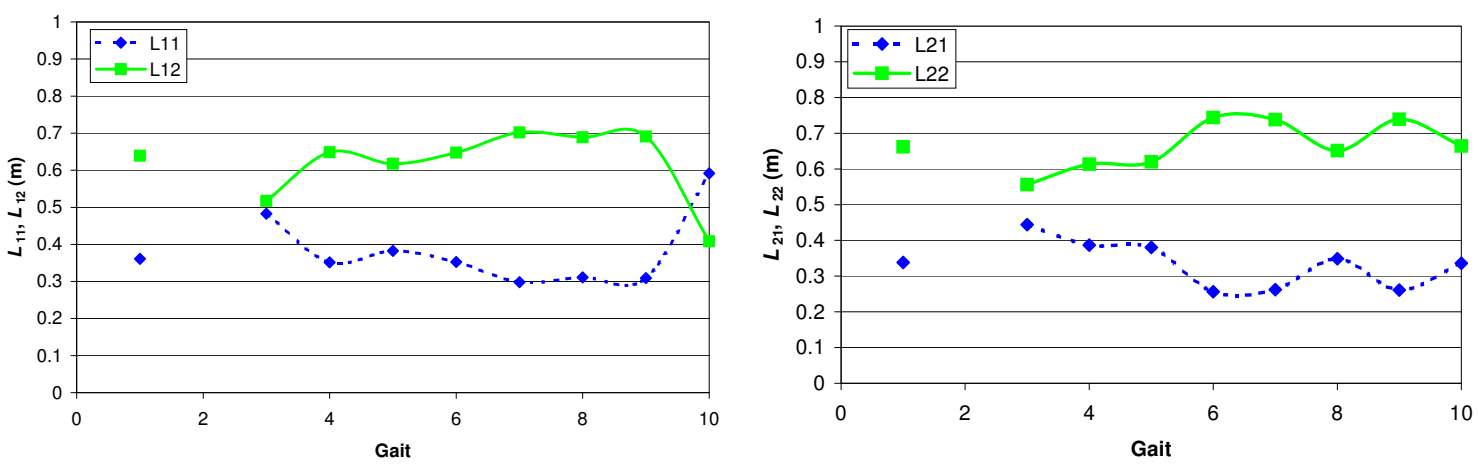

Figure 3: Optimum values of the front legs links lengths $L_{11}$ and $L_{12}$ (left) and of the rear legs links lengths $L_{21}$ and $L_{22}$ (right), for the gaits under study

Figure 3 depicts two charts with the optimum values of the front legs links lengths $L_{11}$ and $L_{12}$ (left) and of the rear legs links lengths $L_{21}$ and $L_{22}$ (right), for the distinct gaits under study, determined by the GA. Analyzing the results presented in these figures it should be referred that the length of the upper segment of the leg should be smaller than the corresponding length of the lower segment. The relation between the lengths of both segments is $L_{i 1} / L_{i 2} \approx 0.3 / 0.7$.

There are only two exceptions to these general results; for the case of the front legs, when the robots adopts the Amble gait both segments should be of similar lengths and when the quadruped adopts the Bound gait the results obtained by the GA indicate that the lower segment of the leg should be smaller than the corresponding upper segment.

In Figure 4 are presented two charts with the optimum values of the foot trajectory offset $O_{1}$ and $\mathrm{O}_{2}$ (left) and of the Body Mass $\left(M_{B}\right)$ (right), determined by the GA, for the gaits under study. Concerning the foot trajectory offset, the results presented in this chart indicate that the robot should move with the feet trajectory displaced to the rear of the hip trajectory (in the moving direction), as indicated by the values of the parameters $O_{i}$. Regarding the robot mass distribution, the body should concentrate most of its value (it is assumed that the total mass of the robot is equal to $100.0 \mathrm{~kg}$ ) being $M_{B}>70 \mathrm{~kg}$ for all gaits under study, except for the Half-Bound.

Finally, Figure 5 shows two charts with the optimum values of the front legs link masses $M_{11}$ and $M_{12}$ (left) and of the rear legs link masses $M_{21}$ and $M_{22}$ (right), determined by the GA, for the gaits under study. The left chart indicates that the lower segment of the front legs should be heavier than the upper segment $\left(M_{12}>M_{11}\right)$, except for the Half-Bound gait.

In a similar manner, the right chart indicates that the lower segment of the rear legs should be 

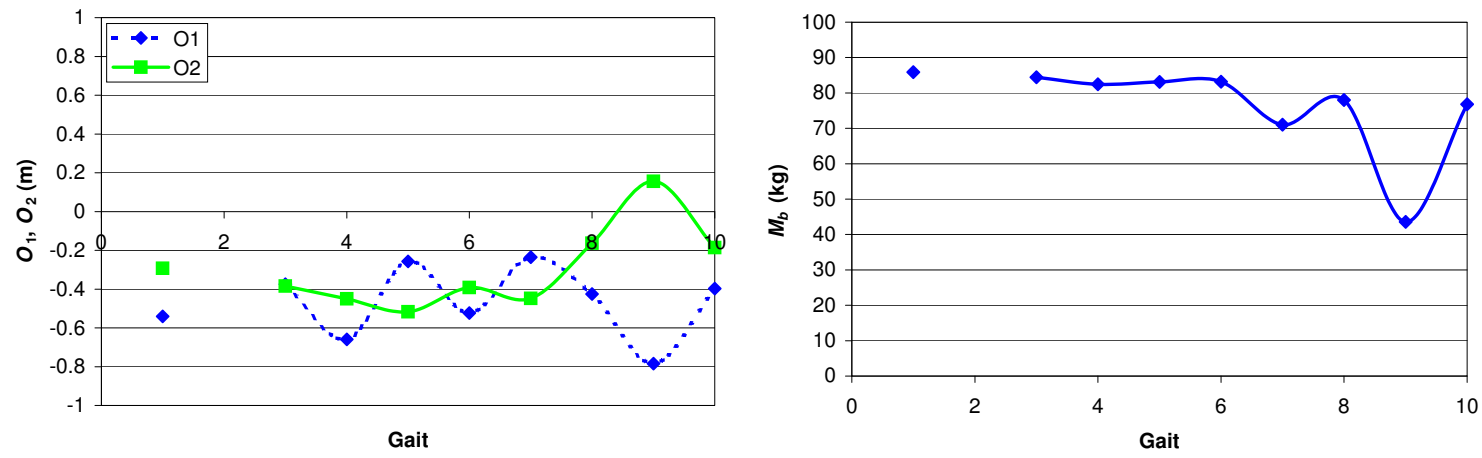

Figure 4: Optimum values of the foot trajectory offset $O_{1}$ and $O_{2}$ (left) and of the Body Mass $M_{B}$ (right), for the gaits under study
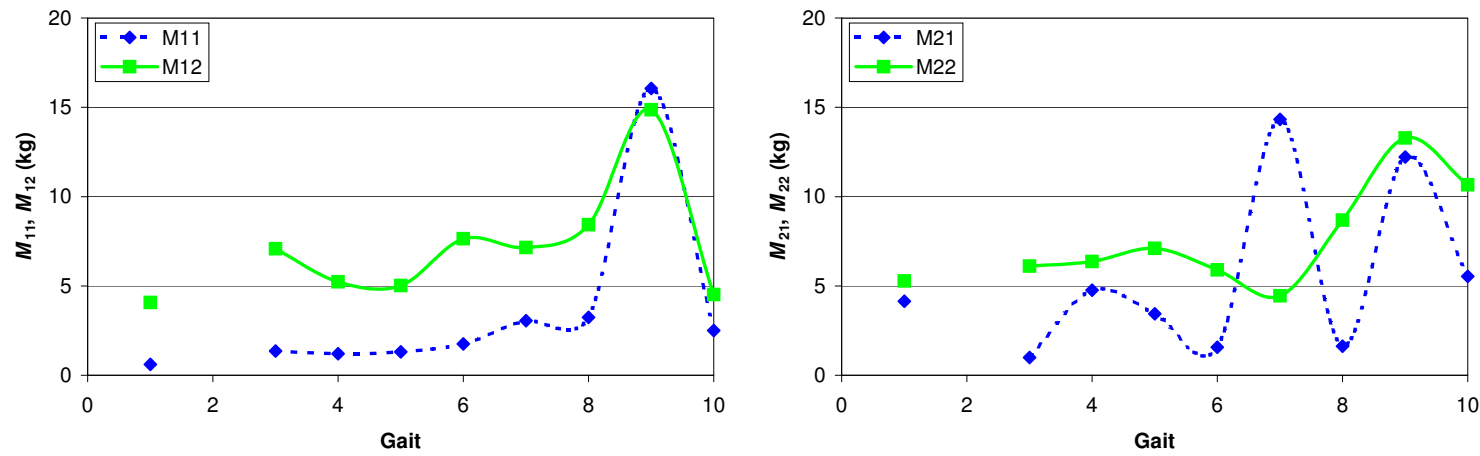

Figure 5: Optimum values of the front legs link masses $M_{11}$ and $M_{12}$ (left) and of the rear legs link masses $M_{21}$ and $M_{22}$ (right), for the gaits under study

heavier than the upper segment $\left(M_{22}>M_{21}\right)$, except for the Transverse Gallop gait. These results seem to agree with the ones presented in Figure 3, since longer legs links segments are heavier.

\section{Conclusions}

This paper presented a GA developed for the optimization of quadruped robot parameters. This GA runs over a simulation application of legged robots (developed in the $\mathrm{C}$ programming language), which allows the optimization of several parameters of the robot model and of its gaits for different locomotion speeds.

Based on this GA, were determined the optimum locomotion parameters for the quadruped robot and its controller, while the robot is moving at $V_{F}=1 \mathrm{~ms}^{-1}$ with distinct gaits.

As ideas for future work, the author plans to develop several simulation experiments to find the parameters that optimize the robot locomotion, from the viewpoint of the index $E_{a v}$, for different values of $V_{F}$ in the range $0.1 \leq V_{F} \leq 10.0 \mathrm{~ms}^{-1}$.

\section{Acknowledgments}

To Sérgio Carvalho, for implementing the basic structure of the GA used in this work. 


\section{References}

[1] M. F. Silva and J. A. T. Machado. A historical perspective of legged robots. J. of Vibration and Control, 13(9-10):1447-1486, 2007.

[2] M. F. Silva and J. A. Tenreiro Machado. A literature review on the optimization of legged robots. J. of Vibration and Control, Accepted for Publication.

[3] S. Farritor, S. Dubowsky, N. Rutman, and J. Cole. A systems-level modular design approach to field robotics. In Proc. of the IEEE Int. Conf. on Rob. and Aut., pages 2890-2895, 1996.

[4] C. Leger. DARWIN2K - An Evolutionary Approach to Automated Design for Robotics. Kluwer Academic Publishers, 2000.

[5] S. Nolfi and D. Floreano. Evolutionary Robotics - The Biology, Intelligence, and Technology of SelfOrganizing Machines. The MIT Press, 2000.

[6] J. Jurez-Guerrero, Muoz-Gutirrez, and W. W. M. Cuevas. Design of a walking machine structure using evolutionary strategies. In Proc. of the IEEE Int. Conf. on Systems, Man and Cybernetics, pages 1427-1432, 1998.

[7] A. Ishiguro, K. Kawasumi, and A. Fujii. Increasing evolvability of a locomotion controller using a passive-dynamic-walking embodiment. In Proc. of the IEEE/RSJ Int. Conf. on Intel. Robots and Systems, pages 2581-2586, 2002.

[8] H. Lipson and J. B. Pollack. Towards continuously reconfigurable self-designing robots. In Proc. of the IEEE Int. Conf. on Rob. and Aut., pages 1761-1766, 2000.

[9] K. Endo, F. Yamasaki, T. Maeno, and H. Kitano. A method for co-evolving morphology and walking pattern of biped humanoid robot. In Proc. of the IEEE Int. Conf. on Rob. and Aut., pages 2775-2780, 2002.

[10] K. Endo, T. Maeno, and H. Kitano. Co-evolution of morphology and walking pattern of biped humanoid robot using evolutionary computation - consideration of characteristic of the servomotors. In Proc. of the IEEE/RSJ Int. Conf. on Intel. Robots and Systems, pages 2678-2683, 2002.

[11] Daniel Marbach and Auke Jan Ijspeert. Co-evolution of configuration and control for homogenous modular robots. In Proc. of IAS'2004 - $8^{\text {th }}$ Conf. on Intelligent Autonomous Systems, pages 712-719, Amsterdam, The Netherlands, March, 10 - 132004.

[12] Geoffrey A. Hollinger and Jeri M. Briscoe. Genetic optimization and simulation of a piezoelectric pipe-crawling inspection robot. In Proc. of the IEEE Int. Conf. on Rob. and Aut., pages 486-491, Barcelona, Spain, April 2005.

[13] M. F. Silva, J. A. T. Machado, and A. M. Lopes. Modelling and simulation of artificial locomotion systems. ROBOTICA, 23(5):595-606, 2005.

[14] S.-M. Song and K.J. Waldron. Machines that Walk: The Adaptive Suspension Vehicle. MIT Press, 1989.

[15] M. F. Silva, J. A. T. Machado, and A. M. Lopes. Position / force control of a walking robot. Machine Intelligence and Robotic Control, 5(2):33-44, 2003.

[16] P. J. Fleming and R. C. Purshouse. Genetic algorithms in control systems engineering. IFAC Professional Brief, 2002.

[17] M. F. Silva and J. A. Tenreiro Machado. Kinematic and dynamic performance analysis of artificial legged systems. ROBOTICA, 26(1):19-39, January 2008. 